\title{
The Insphere of a Tetrahedron
}

\author{
Peter Paul Klein \\ Computing Center, University of Technology Clausthal, Clausthal-Zellerfeld, Germany \\ Email: peter.p.klein@t-online.de
}

How to cite this paper: Klein, P.P. (2020) The Insphere of a Tetrahedron. Applied Mathematics, 11, 601-612.

https://doi.org/10.4236/am.2020.117041

Received: May 14, 2020

Accepted: July 17, 2020

Published: July 20, 2020

Copyright (C) 2020 by author(s) and Scientific Research Publishing Inc. This work is licensed under the Creative Commons Attribution International License (CC BY 4.0).

http://creativecommons.org/licenses/by/4.0/

\begin{abstract}
A contiguous derivation of radius and center of the insphere of a general tetrahedron is given. Therefore a linear system is derived. After a transformation of it the calculation of radius and center can be separated from each other. The remaining linear system for the center of the insphere can be solved after discovering the inverse of the corresponding coefficient matrix. This procedure can also be applied in the planar case to determine radius and center of the incircle of a triangle.
\end{abstract}

\section{Keywords}

Corkscrew Rule, Hesse Normal Form, Decomposed Linear System, Block Upper Triangular Form of Coefficient Matrix, Cofactor Matrix

\section{Introduction}

A coherent derivation of radius and center of the insphere of a general tetrahedron is hard to find (see e.g. [1]). When setting up the linear system for their calculation the uniform orientation of the faces of the tetrahedron has to be observed. This is exemplified in the examples at the end. After transforming the original linear system into a system having a coefficient matrix of block upper triangular form the calculation of radius and center of the insphere of the tetrahedron can be separated from each other. The solution of the remaining linear system for the center of the insphere rests upon an exercise in [2].

\section{Preliminaries}

Matrices are designated by capital letters, such as $B, C, D, E$. The center of the insphere shall be denoted by $P_{m}=\left(x_{m}, y_{m}, z_{m}\right)$ and its radius by $r$. Furthermore the four vertices of the tetrahedron are designated by $P_{i}=\left(x_{i}, y_{i}, z_{i}\right)$ for $i=1,2,3,4$. The four faces of the tetrahedron are triangles having the three vertices: $\left\{P_{1}, P_{2}, P_{3}\right\},\left\{P_{1}, P_{4}, P_{2}\right\},\left\{P_{1}, P_{3}, P_{4}\right\},\left\{P_{2}, P_{4}, P_{3}\right\}$. The order of the vertices 
for each triangle is chosen in such a way, that the normal to a face is directed into the interior of the tetrahedron according to the corkscrew rule: the direction of the normal of a face is defined such that the right thumb points in the direction of the normal and the fingers curl along the orientation of the bounding curve of a triangle.

The plane through the three vertices $P_{1}, P_{2}, P_{3}$ can be described by (see e.g. [3]):

$$
\left|\begin{array}{ccc}
x-x_{1} & y-y_{1} & z-z_{1} \\
x_{2}-x_{1} & y_{2}-y_{1} & z_{2}-z_{1} \\
x_{3}-x_{1} & y_{3}-y_{1} & z_{3}-z_{1}
\end{array}\right|=0 .
$$

Expanding determinant (1) in terms of the elements of the first row leads to:

$$
\left(x-x_{1}\right) d_{123}(y, z)-\left(y-y_{1}\right) d_{123}(x, z)+\left(z-z_{1}\right) d_{123}(x, y)=0
$$

with

$$
\begin{gathered}
d_{123}(y, z)=\left|\begin{array}{ll}
y_{2}-y_{1} & z_{2}-z_{1} \\
y_{3}-y_{1} & z_{3}-z_{1}
\end{array}\right|, \quad d_{123}(x, z)=\left|\begin{array}{ll}
x_{2}-x_{1} & z_{2}-z_{1} \\
x_{3}-x_{1} & z_{3}-z_{1}
\end{array}\right| \\
d_{123}(x, y)=\left|\begin{array}{ll}
x_{2}-x_{1} & y_{2}-y_{1} \\
x_{3}-x_{1} & y_{3}-y_{1}
\end{array}\right|
\end{gathered}
$$

If the vertices $P_{1}, P_{2}, P_{3}$ do not lie on a straight line at least one of the terms $d_{123}(y, z), d_{123}(x, z), d_{123}(x, y)$ is different from zero and so is

$$
w_{123}=\sqrt{d_{123}(y, z)^{2}+d_{123}(x, z)^{2}+d_{123}(x, y)^{2}} .
$$

Dividing Equation (2) by $w_{123}$ leads to the so-called Hesse normal form of the plane

$$
\left(x-x_{1}\right) \frac{d_{123}(y, z)}{w_{123}}-\left(y-y_{1}\right) \frac{d_{123}(x, z)}{w_{123}}+\left(z-z_{1}\right) \frac{d_{123}(x, y)}{w_{123}}=0 .
$$

With the Hesse normal form (5) the distance $r$ of the point $P_{m}=\left(x_{m}, y_{m}, z_{m}\right)$ from the plane can be expressed by (see e.g. [3]):

$$
\left(x_{m}-x_{1}\right) \frac{d_{123}(y, z)}{w_{123}}-\left(y_{m}-y_{1}\right) \frac{d_{123}(x, z)}{w_{123}}+\left(z_{m}-z_{1}\right) \frac{d_{123}(x, y)}{w_{123}}=r .
$$

An illustration is given in Figure 1.

Repeating the above procedure for the plane through the vertices $P_{1}, P_{4}, P_{2}$ of the tetrahedron gives by exchanging the indices $2 \rightarrow 4,3 \rightarrow 2$ :

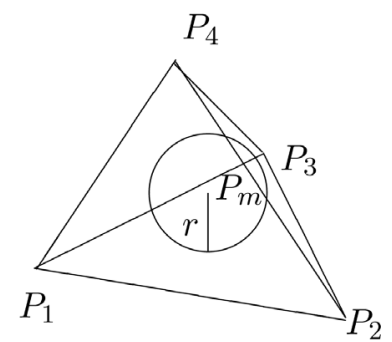

Figure 1. Tetrahedron with insphere. 


$$
\left(x_{m}-x_{1}\right) \frac{d_{142}(y, z)}{w_{142}}-\left(y_{m}-y_{1}\right) \frac{d_{142}(x, z)}{w_{142}}+\left(z_{m}-z_{1}\right) \frac{d_{142}(x, y)}{w_{142}}=r
$$

with

$$
\begin{gathered}
d_{142}(y, z)=\left|\begin{array}{ll}
y_{4}-y_{1} & z_{4}-z_{1} \\
y_{2}-y_{1} & z_{2}-z_{1}
\end{array}\right|, \quad d_{142}(x, z)=\left|\begin{array}{ll}
x_{4}-x_{1} & z_{4}-z_{1} \\
x_{2}-x_{1} & z_{2}-z_{1}
\end{array}\right| \\
d_{142}(x, y)=\left|\begin{array}{ll}
x_{4}-x_{1} & y_{4}-y_{1} \\
x_{2}-x_{1} & y_{2}-y_{1}
\end{array}\right|
\end{gathered}
$$

and

$$
w_{142}=\sqrt{d_{142}(y, z)^{2}+d_{142}(x, z)^{2}+d_{142}(x, y)^{2}} .
$$

Repeating the above procedure for the plane through the vertices $P_{1}, P_{3}, P_{4}$ of the tetrahedron gives by exchanging the indices $4 \rightarrow 3,2 \rightarrow 4$ :

$$
\left(x_{m}-x_{1}\right) \frac{d_{134}(y, z)}{w_{134}}-\left(y_{m}-y_{1}\right) \frac{d_{134}(x, z)}{w_{134}}+\left(z_{m}-z_{1}\right) \frac{d_{134}(x, y)}{w_{134}}=r
$$

with

$$
\begin{gathered}
d_{134}(y, z)=\left|\begin{array}{ll}
y_{3}-y_{1} & z_{3}-z_{1} \\
y_{4}-y_{1} & z_{4}-z_{1}
\end{array}\right|, \quad d_{134}(x, z)=\left|\begin{array}{ll}
x_{3}-x_{1} & z_{3}-z_{1} \\
x_{4}-x_{1} & z_{4}-z_{1}
\end{array}\right| \\
d_{134}(x, y)=\left|\begin{array}{ll}
x_{3}-x_{1} & y_{3}-y_{1} \\
x_{4}-x_{1} & y_{4}-y_{1}
\end{array}\right|
\end{gathered}
$$

and

$$
w_{134}=\sqrt{d_{134}(y, z)^{2}+d_{134}(x, z)^{2}+d_{134}(x, y)^{2}} .
$$

Repeating the above procedure for the plane through the vertices $P_{2}, P_{4}, P_{3}$ of the tetrahedron gives by exchanging the indices $1 \rightarrow 2,3 \rightarrow 4$ and $4 \rightarrow 3$ :

$$
\left(x_{m}-x_{2}\right) \frac{d_{243}(y, z)}{w_{243}}-\left(y_{m}-y_{2}\right) \frac{d_{243}(x, z)}{w_{243}}+\left(z_{m}-z_{2}\right) \frac{d_{243}(x, y)}{w_{243}}=r
$$

with

$$
\begin{gathered}
d_{243}(y, z)=\left|\begin{array}{ll}
y_{4}-y_{2} & z_{4}-z_{2} \\
y_{3}-y_{2} & z_{3}-z_{2}
\end{array}\right|, \quad d_{243}(x, z)=\left|\begin{array}{ll}
x_{4}-x_{2} & z_{4}-z_{2} \\
x_{3}-x_{2} & z_{3}-z_{2}
\end{array}\right| \\
d_{243}(x, y)=\left|\begin{array}{ll}
x_{4}-x_{2} & y_{4}-y_{2} \\
x_{3}-x_{2} & y_{3}-y_{2}
\end{array}\right|
\end{gathered}
$$

and

$$
w_{243}=\sqrt{d_{243}(y, z)^{2}+d_{243}(x, z)^{2}+d_{243}(x, y)^{2}} .
$$

By splitting the terms $x_{m}-x_{2}, y_{m}-y_{2}, z_{m}-z_{2}$ into

$$
x_{m}-x_{1}-\left(x_{2}-x_{1}\right), \quad y_{m}-y_{1}-\left(y_{2}-y_{1}\right), \quad z_{m}-z_{1}-\left(z_{2}-z_{1}\right)
$$

one obtains from (13)

$$
\left(x_{m}-x_{1}\right) \frac{d_{243}(y, z)}{w_{243}}-\left(y_{m}-y_{1}\right) \frac{d_{243}(x, z)}{w_{243}}+\left(z_{m}-z_{1}\right) \frac{d_{243}(x, y)}{w_{243}}+\frac{\rho}{w_{243}}=r
$$

with 


$$
\rho=-\left(\left(x_{2}-x_{1}\right) d_{243}(y, z)-\left(y_{2}-y_{1}\right) d_{243}(x, z)+\left(z_{2}-z_{1}\right) d_{243}(x, y)\right) ;
$$

$\rho$ can also be written as a determinant of the following matrix

$$
C=\left(\begin{array}{lll}
x_{2}-x_{1} & y_{2}-y_{1} & z_{2}-z_{1} \\
x_{3}-x_{1} & y_{3}-y_{1} & z_{3}-z_{1} \\
x_{4}-x_{1} & y_{4}-y_{1} & z_{4}-z_{1}
\end{array}\right) .
$$

From determinant $|C|$ one obtains (17) by subtracting the first row from the second and third row, exchanging rows two and three and expanding the resulting determinant with respect to the first row. This process is reversible.

\section{Corollary 1}

For determinant $|C|$ holds $|C|=0$ if and only if the four vertices $P_{1}, P_{2}, P_{3}$, $P_{4}$ of the tetrahedron are lying in a plane.

Proof: Considering for instance the plane through $P_{1}, P_{2}, P_{3}$ according to (1) the coordinates of vertex $P_{4}$ are fulfilling (1) if and only if determinant $|C|$ is zero after exchanging the third row with the second and afterwards with the first row, leaving the value of the determinant unchanged.

\section{A Linear System}

From Equations (6), (7), (10) and (16) one obtains the following system of linear equations for the unknowns $x_{m}-x_{1}, y_{m}-y_{1}, z_{m}-z_{1}, r$ :

$$
\left(\begin{array}{cccc}
d_{123}(y, z) & -d_{123}(x, z) & d_{123}(x, y) & -w_{123} \\
d_{142}(y, z) & -d_{142}(x, z) & d_{142}(x, y) & -w_{142} \\
d_{134}(y, z) & -d_{134}(x, z) & d_{134}(x, y) & -w_{134} \\
d_{243}(y, z) & -d_{243}(x, z) & d_{243}(x, y) & -w_{243}
\end{array}\right)\left(\begin{array}{c}
x_{m}-x_{1} \\
y_{m}-y_{1} \\
z_{m}-z_{1} \\
r
\end{array}\right)=\left(\begin{array}{c}
0 \\
0 \\
0 \\
-|C|
\end{array}\right) \text {. }
$$

With the following Proposition the elements $d_{243}(y, z), d_{243}(x, z), d_{243}(x, y)$ in the last row of the linear system (19) can be expressed by the remaining elements.

Proposition 1

$$
\begin{aligned}
& d_{243}(y, z)+d_{134}(y, z)+d_{123}(y, z)+d_{142}(y, z)=0 \\
& d_{243}(x, z)+d_{134}(x, z)+d_{123}(x, z)+d_{142}(x, z)=0 \\
& d_{243}(x, y)+d_{134}(x, y)+d_{123}(x, y)+d_{142}(x, y)=0
\end{aligned}
$$

Proof: It is sufficient to prove the first statement.

$$
\begin{aligned}
d_{243}(y, z) & =\left|\begin{array}{ll}
y_{4}-y_{2} & z_{4}-z_{2} \\
y_{3}-y_{2} & z_{3}-z_{2}
\end{array}\right| \\
& =\left|\begin{array}{ll}
y_{4}-y_{1}-\left(y_{2}-y_{1}\right) & z_{4}-z_{1}-\left(z_{2}-z_{1}\right) \\
y_{3}-y_{1}-\left(y_{2}-y_{1}\right) & z_{3}-z_{1}-\left(z_{2}-z_{1}\right)
\end{array}\right| \\
& =\left|\begin{array}{ll}
y_{4}-y_{1} & z_{4}-z_{1} \\
y_{3}-y_{1} & z_{3}-z_{1}
\end{array}\right|-\left|\begin{array}{ll}
y_{2}-y_{1} & z_{2}-z_{1} \\
y_{3}-y_{1} & z_{3}-z_{1}
\end{array}\right|+\left|\begin{array}{ll}
y_{2}-y_{1} & z_{2}-z_{1} \\
y_{4}-y_{1} & z_{4}-z_{1}
\end{array}\right| \\
& =-\left|\begin{array}{ll}
y_{3}-y_{1} & z_{3}-z_{1} \\
y_{4}-y_{1} & z_{4}-z_{1}
\end{array}\right|-\left|\begin{array}{ll}
y_{2}-y_{1} & z_{2}-z_{1} \\
y_{3}-y_{1} & z_{3}-z_{1}
\end{array}\right|-\left|\begin{array}{ll}
y_{4}-y_{1} & z_{4}-z_{1} \\
y_{2}-y_{1} & z_{2}-z_{1}
\end{array}\right| \\
& =-d_{134}(y, z)-d_{123}(y, z)-d_{142}(y, z) .
\end{aligned}
$$


Thus

$$
d_{243}(y, z)+d_{134}(y, z)+d_{123}(y, z)+d_{142}(y, z)=0 .
$$

Multiplying the linear system (19) from the left by the following transformation matrix

$$
\left(\begin{array}{llll}
1 & 0 & 0 & 0 \\
0 & 1 & 0 & 0 \\
0 & 0 & 1 & 0 \\
1 & 1 & 1 & 1
\end{array}\right)
$$

-or what is equivalent, by adding the first three rows of (19) to the last row-yields with the result of Proposition 1 a decomposed linear system, whose coefficient matrix has block upper triangular form

$$
\left(\begin{array}{cccc}
d_{123}(y, z) & -d_{123}(x, z) & d_{123}(x, y) & -w_{123} \\
d_{142}(y, z) & -d_{142}(x, z) & d_{142}(x, y) & -w_{142} \\
d_{134}(y, z) & -d_{134}(x, z) & d_{134}(x, y) & -w_{134} \\
0 & 0 & 0 & -\sum w_{i j k}
\end{array}\right)\left(\begin{array}{c}
x_{m}-x_{1} \\
y_{m}-y_{1} \\
z_{m}-z_{1} \\
r
\end{array}\right)=\left(\begin{array}{c}
0 \\
0 \\
0 \\
-|C|
\end{array}\right) .
$$

The summation of the terms $w_{i j k}$ in the last row of (20) ranges over the index triplets 123,142, 134 and 243. Looking at the last equation of (20) the calculation of $r$ can be separated from the calculation of the other unknowns. The last equation of (20) yields:

$$
r=\frac{|C|}{w_{123}+w_{142}+w_{134}+w_{243}},
$$

where each of the terms $w_{123}, w_{142}, w_{134}$ and $w_{243}$ according to (4), (9), (12) and (15) is non-zero, if the three vertices involved do not lie on straight lines. Thus one obtains from (20) a linear system for the remaining unknowns $x_{m}-x_{1}$, $y_{m}-y_{1}, z_{m}-z_{1}$. For convenience the first and the third row of (20) are interchanged.

$$
\left(\begin{array}{lll}
d_{134}(y, z) & -d_{134}(x, z) & d_{134}(x, y) \\
d_{142}(y, z) & -d_{142}(x, z) & d_{142}(x, y) \\
d_{123}(y, z) & -d_{123}(x, z) & d_{123}(x, y)
\end{array}\right)\left(\begin{array}{c}
x_{m}-x_{1} \\
y_{m}-y_{1} \\
z_{m}-z_{1}
\end{array}\right)=\left(\begin{array}{l}
r w_{134} \\
r w_{142} \\
r w_{123}
\end{array}\right)
$$

The next Proposition is formulated for the coefficient matrix

$$
D=\left(\begin{array}{lll}
d_{134}(y, z) & -d_{134}(x, z) & d_{134}(x, y) \\
d_{142}(y, z) & -d_{142}(x, z) & d_{142}(x, y) \\
d_{123}(y, z) & -d_{123}(x, z) & d_{123}(x, y)
\end{array}\right)
$$

of the linear system (22). This Proposition rests upon an exercise in [2].

\section{Proposition 2}

For matrix $C$ from (18) and matrix $D$ from (23) the following statement is true: $C^{\mathrm{T}} D=|C| E$, where $C^{\mathrm{T}}$ denotes the transpose of $C$ and $E$ the identity matrix. This means that $D$ is the cofactor matrix of $C$. Furthermore for $|C| \neq 0$ holds: $\frac{1}{|C|} C^{\mathrm{T}}$ is the inverse of $D$ and $|D|=|C|^{2}$. 
Proof: Expanding $|C|$ in terms of the elements of the first, second and third column yields with denotations (11), (8), (3):

$$
\begin{aligned}
& |C|=\left(x_{2}-x_{1}\right)\left|\begin{array}{ll}
y_{3}-y_{1} & z_{3}-z_{1} \\
y_{4}-y_{1} & z_{4}-z_{1}
\end{array}\right|-\left(x_{3}-x_{1}\right)\left|\begin{array}{ll}
y_{2}-y_{1} & z_{2}-z_{1} \\
y_{4}-y_{1} & z_{4}-z_{1}
\end{array}\right| \\
& +\left(x_{4}-x_{1}\right)\left|\begin{array}{ll}
y_{2}-y_{1} & z_{2}-z_{1} \\
y_{3}-y_{1} & z_{3}-z_{1}
\end{array}\right| \\
& =\left(x_{2}-x_{1}\right) d_{134}(y, z)+\left(x_{3}-x_{1}\right) d_{142}(y, z)+\left(x_{4}-x_{1}\right) d_{123}(y, z) \\
& |C|=-\left(y_{2}-y_{1}\right)\left|\begin{array}{ll}
x_{3}-x_{1} & z_{3}-z_{1} \\
x_{4}-x_{1} & z_{4}-z_{1}
\end{array}\right|+\left(y_{3}-y_{1}\right)\left|\begin{array}{ll}
x_{2}-x_{1} & z_{2}-z_{1} \\
x_{4}-x_{1} & z_{4}-z_{1}
\end{array}\right| \\
& -\left(y_{4}-y_{1}\right)\left|\begin{array}{ll}
x_{2}-x_{1} & z_{2}-z_{1} \\
x_{3}-x_{1} & z_{3}-z_{1}
\end{array}\right| \\
& =-\left(y_{2}-y_{1}\right) d_{134}(x, z)-\left(y_{3}-y_{1}\right) d_{142}(x, z)-\left(y_{4}-y_{1}\right) d_{123}(x, z) \\
& |C|=\left(z_{2}-z_{1}\right)\left|\begin{array}{ll}
x_{3}-x_{1} & y_{3}-y_{1} \\
x_{4}-x_{1} & y_{4}-y_{1}
\end{array}\right|-\left(z_{3}-z_{1}\right)\left|\begin{array}{ll}
x_{2}-x_{1} & y_{2}-y_{1} \\
x_{4}-x_{1} & y_{4}-y_{1}
\end{array}\right| \\
& +\left(z_{4}-z_{1}\right)\left|\begin{array}{ll}
x_{2}-x_{1} & y_{2}-y_{1} \\
x_{3}-x_{1} & y_{3}-y_{1}
\end{array}\right| \\
& =\left(z_{2}-z_{1}\right) d_{134}(x, y)+\left(z_{3}-z_{1}\right) d_{142}(x, y)+\left(z_{4}-z_{1}\right) d_{123}(x, y)
\end{aligned}
$$

By multiplication of matrices $C^{\mathrm{T}}$ and $D$ one obtains with the above results for determinant $|C|$ the elements on the main diagonal of the product matrix:

$$
\begin{aligned}
C^{\mathrm{T}} D & =\left(\begin{array}{ccc}
x_{2}-x_{1} & x_{3}-x_{1} & x_{4}-x_{1} \\
y_{2}-y_{1} & y_{3}-y_{1} & y_{4}-y_{1} \\
z_{2}-z_{1} & z_{3}-z_{1} & z_{4}-z_{1}
\end{array}\right)\left(\begin{array}{lll}
d_{134}(y, z) & -d_{134}(x, z) & d_{134}(x, y) \\
d_{142}(y, z) & -d_{142}(x, z) & d_{142}(x, y) \\
d_{123}(y, z) & -d_{123}(x, z) & d_{123}(x, y)
\end{array}\right) \\
& =\left(\begin{array}{ccc}
|C| & 0 & 0 \\
0 & |C| & 0 \\
0 & 0 & |C|
\end{array}\right) .
\end{aligned}
$$

The non-diagonal elements of the product matrix are zero because they can be written as $3 \times 3$ determinants with two equal columns. As an example this shall be shown for the element in row two and column one of the product matrix:

$$
\begin{aligned}
& \left(y_{2}-y_{1}\right) d_{134}(y, z)+\left(y_{3}-y_{1}\right) d_{142}(y, z)+\left(y_{4}-y_{1}\right) d_{123}(y, z) \\
= & \left(y_{2}-y_{1}\right)\left|\begin{array}{ll}
y_{3}-y_{1} & z_{3}-z_{1} \\
y_{4}-y_{1} & z_{4}-z_{1}
\end{array}\right|-\left(y_{3}-y_{1}\right)\left|\begin{array}{ll}
y_{2}-y_{1} & z_{2}-z_{1} \\
y_{4}-y_{1} & z_{4}-z_{1}
\end{array}\right| \\
& +\left(y_{4}-y_{1}\right)\left|\begin{array}{ll}
y_{2}-y_{1} & z_{2}-z_{1} \\
y_{3}-y_{1} & z_{3}-z_{1}
\end{array}\right| \\
= & \left|\begin{array}{lll}
y_{2}-y_{1} & y_{2}-y_{1} & z_{2}-z_{1} \\
y_{3}-y_{1} & y_{3}-y_{1} & z_{3}-z_{1} \\
y_{4}-y_{1} & y_{4}-y_{1} & z_{4}-z_{1}
\end{array}\right|=0
\end{aligned}
$$

From (24) follows in case of $|C| \neq 0$ that $\frac{1}{|C|} C^{\mathrm{T}}$ is the inverse of $D$. Forming determinants in (24) yields $\left|C^{\mathrm{T}}\right||D|=|C|^{3}$. Because of $\left|C^{\mathrm{T}}\right|=|C|$ for $|C| \neq 0$ one obtains $|D|=|C|^{2}$. 


\section{Corollary 2}

If for determinant $|C|$ holds $|C| \neq 0$ the coefficient matrix $D$ of the linear system (22) has the following determinant

$$
|D|=\left|\begin{array}{llll}
x_{1} & y_{1} & z_{1} & 1 \\
x_{2} & y_{2} & z_{2} & 1 \\
x_{3} & y_{3} & z_{3} & 1 \\
x_{4} & y_{4} & z_{4} & 1
\end{array}\right|^{2} .
$$

Proof: Determinant $|C|$ can be transformed in the following way:

$$
\begin{aligned}
|C| & =\left|\begin{array}{cccc}
x_{1} & y_{1} & z_{1} & -1 \\
x_{2}-x_{1} & y_{2}-y_{1} & z_{2}-z_{1} & 0 \\
x_{3}-x_{1} & y_{3}-y_{1} & z_{3}-z_{1} & 0 \\
x_{4}-x_{1} & y_{4}-y_{1} & z_{4}-z_{1} & 0
\end{array}\right| \\
& =\left|\begin{array}{llll}
x_{1} & y_{1} & z_{1} & -1 \\
x_{2} & y_{2} & z_{2} & -1 \\
x_{3} & y_{3} & z_{3} & -1 \\
x_{4} & y_{4} & z_{4} & -1
\end{array}\right|=-\left|\begin{array}{llll}
x_{1} & y_{1} & z_{1} & 1 \\
x_{2} & y_{2} & z_{2} & 1 \\
x_{3} & y_{3} & z_{3} & 1 \\
x_{4} & y_{4} & z_{4} & 1
\end{array}\right| .
\end{aligned}
$$

With (26) and the result of Proposition 2 for determinant $|D|$ of the linear system (22) holds (25).

Finally the coordinates of the center of the insphere of the tetrahedron are obtained after solving the linear system (22). The result may also be found in [4].

\section{Theorem 1}

If the vertices $P_{1}, P_{2}, P_{3}, P_{4}$ of the tetrahedron are not lying in a plane the center of the insphere is given by:

$$
\begin{aligned}
& x_{m}=\frac{w_{123} x_{4}+w_{142} x_{3}+w_{134} x_{2}+w_{243} x_{1}}{w_{123}+w_{142}+w_{134}+w_{243}} \\
& y_{m}=\frac{w_{123} y_{4}+w_{142} y_{3}+w_{134} y_{2}+w_{243} y_{1}}{w_{123}+w_{142}+w_{134}+w_{243}} \\
& z_{m}=\frac{w_{123} z_{4}+w_{142} z_{3}+w_{134} z_{2}+w_{243} z_{1}}{w_{123}+w_{142}+w_{134}+w_{243}} .
\end{aligned}
$$

Proof: The three vertices of each face of the tetrahedron cannot lie on a straight line; otherwise the vertices $P_{1}, P_{2}, P_{3}, P_{4}$ would lie in a plane. So the terms $w_{123}, w_{142}, w_{134}, w_{243}$ defined in (4), (9), (12) and (15) are different from zero. Furthermore according to Corollary 1 determinant $|C|$ is different from zero.

Multiplying the linear system (22) from the left by $\frac{1}{|C|} C^{\mathrm{T}}$, the inverse of coefficient matrix D according to Proposition 2, yields

$$
\begin{aligned}
& x_{m}-x_{1}=\frac{r}{|C|}\left(w_{134}\left(x_{2}-x_{1}\right)+w_{142}\left(x_{3}-x_{1}\right)+w_{123}\left(x_{4}-x_{1}\right)\right), \\
& y_{m}-y_{1}=\frac{r}{|C|}\left(w_{134}\left(y_{2}-y_{1}\right)+w_{142}\left(y_{3}-y_{1}\right)+w_{123}\left(y_{4}-y_{1}\right)\right),
\end{aligned}
$$




$$
z_{m}-z_{1}=\frac{r}{|C|}\left(w_{134}\left(z_{2}-z_{1}\right)+w_{142}\left(z_{3}-z_{1}\right)+w_{123}\left(z_{4}-z_{1}\right)\right) .
$$

With formula (21) for $r$ one obtains

$$
\begin{aligned}
& x_{m}-x_{1}=\frac{w_{134}\left(x_{2}-x_{1}\right)+w_{142}\left(x_{3}-x_{1}\right)+w_{123}\left(x_{4}-x_{1}\right)}{w_{123}+w_{142}+w_{134}+w_{243}} . \\
& y_{m}-y_{1}=\frac{w_{134}\left(y_{2}-y_{1}\right)+w_{142}\left(y_{3}-y_{1}\right)+w_{123}\left(y_{4}-y_{1}\right)}{w_{123}+w_{142}+w_{134}+w_{243}} . \\
& z_{m}-z_{1}=\frac{w_{134}\left(z_{2}-z_{1}\right)+w_{142}\left(z_{3}-z_{1}\right)+w_{123}\left(z_{4}-z_{1}\right)}{w_{123}+w_{142}+w_{134}+w_{243}} .
\end{aligned}
$$

From (30), (31), (32) one can deduce (27), (28), (29).

\section{The Planar Case}

Assume that the three vertices of a triangle $P_{1}=\left(x_{1}, y_{1}\right), P_{2}=\left(x_{2}, y_{2}\right)$, $P_{3}=\left(x_{3}, y_{3}\right)$ are not coinciding. The center of the incircle of the triangle shall be denoted by $P_{m}=\left(x_{m}, y_{m}\right)$ and its radius by $r$.

The straight line through $P_{1}$ and $P_{2}$ is given by

$$
\left|\begin{array}{cc}
x-x_{1} & y-y_{1} \\
x_{2}-x_{1} & y_{2}-y_{1}
\end{array}\right|=\left(x-x_{1}\right)\left(y_{2}-y_{1}\right)-\left(y-y_{1}\right)\left(x_{2}-x_{1}\right)=0 .
$$

The so-called Hesse normal form of the line is obtained by dividing the equation by

$$
w_{12}=\sqrt{\left(x_{2}-x_{1}\right)^{2}+\left(y_{2}-y_{1}\right)^{2}}
$$

yielding

$$
\left(x-x_{1}\right) \frac{y_{2}-y_{1}}{w_{12}}-\left(y-y_{1}\right) \frac{x_{2}-x_{1}}{w_{12}}=0 .
$$

The distance $r$ of the point $P_{m}=\left(x_{m}, y_{m}\right)$ from this line is given by (see e.g. [3])

$$
\left(x_{m}-x_{1}\right) \frac{y_{2}-y_{1}}{w_{12}}-\left(y_{m}-y_{1}\right) \frac{x_{2}-x_{1}}{w_{12}}=r .
$$

Repeating the above procedure for the line through $P_{2}$ and $P_{3}$ of the triangle gives by exchanging the indices $1 \rightarrow 2$ and $2 \rightarrow 3$

$$
\left(x_{m}-x_{2}\right) \frac{y_{3}-y_{2}}{w_{23}}-\left(y_{m}-y_{2}\right) \frac{x_{3}-x_{2}}{w_{23}}=r
$$

with

$$
w_{23}=\sqrt{\left(x_{3}-x_{2}\right)^{2}+\left(y_{3}-y_{2}\right)^{2}} .
$$

By splitting the terms $x_{m}-x_{2}, y_{m}-y_{2}$ into

$$
x_{m}-x_{1}-\left(x_{2}-x_{1}\right), \quad y_{m}-y_{1}-\left(y_{2}-y_{1}\right)
$$

one obtains 


$$
\left(x_{m}-x_{1}\right) \frac{y_{3}-y_{2}}{w_{23}}-\left(y_{m}-y_{1}\right) \frac{x_{3}-x_{2}}{w_{23}}+\frac{\sigma}{w_{23}}=r
$$

with

$$
\begin{aligned}
\sigma & =-\left(\left(x_{2}-x_{1}\right)\left(y_{3}-y_{2}\right)-\left(y_{2}-y_{1}\right)\left(x_{3}-x_{2}\right)\right) \\
& =-\left|\begin{array}{ll}
x_{2}-x_{1} & y_{2}-y_{1} \\
x_{3}-x_{2} & y_{3}-y_{2}
\end{array}\right|=-\left|\begin{array}{ll}
x_{2}-x_{1} & y_{2}-y_{1} \\
x_{3}-x_{1} & y_{3}-y_{1}
\end{array}\right| \\
& =\left|\begin{array}{ll}
x_{3}-x_{1} & y_{3}-y_{1} \\
x_{2}-x_{1} & y_{2}-y_{1}
\end{array}\right| .
\end{aligned}
$$

Introducing matrix

$$
B=\left(\begin{array}{ll}
x_{3}-x_{1} & y_{3}-y_{1} \\
x_{2}-x_{1} & y_{2}-y_{1}
\end{array}\right)
$$

and its determinant $|B|$ Equation (34) turns into

$$
\left(x_{m}-x_{1}\right) \frac{y_{3}-y_{2}}{w_{23}}-\left(y_{m}-y_{1}\right) \frac{x_{3}-x_{2}}{w_{23}}+\frac{|B|}{w_{23}}=r .
$$

As the vertices of the triangle were assumed to be not coinciding $|B| \neq 0$ is true. Repeating the above procedure for the line through $P_{3}$ and $P_{1}$ of the triangle gives by exchanging the indices $2 \rightarrow 3$ and $3 \rightarrow 1$

$$
\left(x_{m}-x_{3}\right) \frac{y_{1}-y_{3}}{w_{31}}-\left(y_{m}-y_{3}\right) \frac{x_{1}-x_{3}}{w_{31}}=r
$$

with

$$
w_{31}=\sqrt{\left(x_{1}-x_{3}\right)^{2}+\left(y_{1}-y_{3}\right)^{2}} .
$$

By splitting the terms $x_{m}-x_{3}, y_{m}-y_{3}$ into

$$
x_{m}-x_{1}-\left(x_{3}-x_{1}\right), \quad y_{m}-y_{1}-\left(y_{3}-y_{1}\right)
$$

one obtains

$$
\left(x_{m}-x_{1}\right) \frac{y_{1}-y_{3}}{w_{31}}-\left(y_{m}-y_{1}\right) \frac{x_{1}-x_{3}}{w_{31}}+\frac{\tau}{w_{31}}=r
$$

with

$$
\tau=-\left(\left(x_{3}-x_{1}\right)\left(y_{1}-y_{3}\right)-\left(y_{3}-y_{1}\right)\left(x_{1}-x_{3}\right)\right)=0 .
$$

Equations (33), (36) and (35) are forming a linear system for the unknowns $x_{m}-x_{1}, y_{m}-y_{1}$ and $r$.

$$
\left(\begin{array}{lll}
y_{2}-y_{1} & -\left(x_{2}-x_{1}\right) & -w_{12} \\
y_{1}-y_{3} & -\left(x_{1}-x_{3}\right) & -w_{31} \\
y_{3}-y_{2} & -\left(x_{3}-x_{2}\right) & -w_{23}
\end{array}\right)\left(\begin{array}{c}
x_{m}-x_{1} \\
y_{m}-y_{1} \\
r
\end{array}\right)=\left(\begin{array}{c}
0 \\
0 \\
-|B|
\end{array}\right) .
$$

Adding the first and the second row of the linear system (37) to the last row yields

$$
\left(\begin{array}{ccc}
y_{2}-y_{1} & -\left(x_{2}-x_{1}\right) & -w_{12} \\
y_{1}-y_{3} & -\left(x_{1}-x_{3}\right) & -w_{31} \\
0 & 0 & -\sum w_{i j}
\end{array}\right)\left(\begin{array}{c}
x_{m}-x_{1} \\
y_{m}-y_{1} \\
r
\end{array}\right)=\left(\begin{array}{c}
0 \\
0 \\
-|B|
\end{array}\right),
$$


where the sum in the third row of (38) ranges over the index doublets 12, 31 and 23. From the third row of (38) one obtains

$$
r=\frac{|B|}{w_{12}+w_{31}+w_{23}} .
$$

The remaining reduced linear system for $x_{m}-x_{1}$ and $y_{m}-y_{1}$ reads

$$
\left(\begin{array}{ll}
y_{2}-y_{1} & -\left(x_{2}-x_{1}\right) \\
y_{1}-y_{3} & -\left(x_{1}-x_{3}\right)
\end{array}\right)\left(\begin{array}{l}
x_{m}-x_{1} \\
y_{m}-y_{1}
\end{array}\right)=\left(\begin{array}{l}
r w_{12} \\
r w_{31}
\end{array}\right) \text {. }
$$

Multiplying the linear system (40) from the left by $B^{T}$ yields because of

$$
\left(\begin{array}{ll}
x_{3}-x_{1} & x_{2}-x_{1} \\
y_{3}-y_{1} & y_{2}-y_{1}
\end{array}\right)\left(\begin{array}{ll}
y_{2}-y_{1} & -\left(x_{2}-x_{1}\right) \\
y_{1}-y_{3} & -\left(x_{1}-x_{3}\right)
\end{array}\right)=\left(\begin{array}{cc}
|B| & 0 \\
0 & |B|
\end{array}\right)
$$

and $|B| \neq 0$ as solution

$$
\begin{aligned}
& x_{m}-x_{1}=\frac{r}{|B|}\left(w_{12}\left(x_{3}-x_{1}\right)+w_{31}\left(x_{2}-x_{1}\right)\right) \\
& y_{m}-y_{1}=\frac{r}{|B|}\left(w_{12}\left(y_{3}-y_{1}\right)+w_{31}\left(y_{2}-y_{1}\right)\right) .
\end{aligned}
$$

With formula (39) for $r$ one obtains from this result

$$
\begin{aligned}
& x_{m}-x_{1}=\frac{w_{12}\left(x_{3}-x_{1}\right)+w_{31}\left(x_{2}-x_{1}\right)}{w_{12}+w_{31}+w_{23}} \\
& y_{m}-y_{1}=\frac{w_{12}\left(y_{3}-y_{1}\right)+w_{31}\left(y_{2}-y_{1}\right)}{w_{12}+w_{31}+w_{23}}
\end{aligned}
$$

and thus

$$
\begin{gathered}
x_{m}=\frac{w_{12} x_{3}+w_{31} x_{2}+w_{23} x_{1}}{w_{12}+w_{31}+w_{23}} \\
y_{m}=\frac{w_{12} y_{3}+w_{31} y_{2}+w_{23} y_{1}}{w_{12}+w_{31}+w_{23}} .
\end{gathered}
$$

\section{Examples}

The tetrahedrons considered in the sequel are having as vertex the origin, a vertex on the $x$-axis, a vertex in the $x-y$ plane and a vertex in three-dimensional space: $P_{1}=(0,0,0), P_{2}=\left(x_{2}, 0,0\right), P_{3}=\left(x_{3}, y_{3}, 0\right)$ and $P_{4}=\left(x_{4}, y_{4}, z_{4}\right)$. For determinant $|C|$ according to (26) holds:

$$
|C|=-\left|\begin{array}{llll}
0 & 0 & 0 & 1 \\
x_{2} & 0 & 0 & 1 \\
x_{3} & y_{3} & 0 & 1 \\
x_{4} & y_{4} & z_{4} & 1
\end{array}\right|=-\left|\begin{array}{cccc}
0 & 0 & 0 & 1 \\
x_{2} & 0 & 0 & 0 \\
x_{3} & y_{3} & 0 & 0 \\
x_{4} & y_{4} & z_{4} & 0
\end{array}\right|=\left|\begin{array}{ccc}
x_{2} & 0 & 0 \\
x_{3} & y_{3} & 0 \\
x_{4} & y_{4} & z_{4}
\end{array}\right|=x_{2} y_{3} z_{4} .
$$

Determinant $|C|$ is different from zero if the product $x_{2} y_{3} z_{4}$ is different 
from zero.

\section{Example 1}

Consider a regular tetrahedron of edge length $a$, having the vertices $P_{1}=(0,0,0), P_{2}=(a, 0,0), P_{3}=\left(\frac{a}{2}, \frac{\sqrt{3}}{2} a, 0\right)$ and $P_{4}=\left(\frac{a}{2}, \frac{1}{2 \sqrt{3}} a, \sqrt{\frac{2}{3}} a\right)$. For it holds $w_{123}=w_{142}=w_{134}=w_{243}=\frac{\sqrt{3}}{2} a^{2}$ and $|C|=\frac{1}{\sqrt{2}} a^{3}$. From (21) the radius is $r=\frac{1}{2 \sqrt{6}} a$. The linear system (22) for the calculation of the center of the insphere reads:

$$
\left(\begin{array}{ccc}
\frac{1}{\sqrt{2}} a^{2} & -\frac{1}{\sqrt{6}} a^{2} & -\frac{1}{2 \sqrt{3}} a^{2} \\
0 & \sqrt{\frac{2}{3}} a^{2} & -\frac{1}{2 \sqrt{3}} a^{2} \\
0 & 0 & \frac{\sqrt{3}}{2} a^{2}
\end{array}\right)\left(\begin{array}{l}
x_{m} \\
y_{m} \\
z_{m}
\end{array}\right)=\left(\begin{array}{l}
\frac{1}{4 \sqrt{2}} a^{3} \\
\frac{1}{4 \sqrt{2}} a^{3} \\
\frac{1}{4 \sqrt{2}} a^{3}
\end{array}\right) .
$$

The solution of the linear system is: $P_{m}=\left(\frac{a}{2}, \frac{1}{2 \sqrt{3}} a, \frac{1}{2 \sqrt{6}} a\right)$. The same result is obtained with (27), (28), (29). The distance of $P_{m}$ from each vertex is $\frac{1}{2} \sqrt{\frac{3}{2}} a$.

\section{Example 2}

Consider an irregular tetrahedron with the vertices $P_{1}=(0,0,0), P_{2}=(6,0,0)$, $P_{3}=(5,4,0)$ and $P_{4}=(2,3,7) \cdot|C|=168$ and from (21) the radius is $r=1.14508$. The linear system (22) for the calculation of the center of the insphere reads using decimal numbers:

$$
\left(\begin{array}{ccc}
28 & -35 & 7 \\
0 & 42 & -18 \\
0 & 0 & 24
\end{array}\right)\left(\begin{array}{l}
x_{m} \\
y_{m} \\
z_{m}
\end{array}\right)=\left(\begin{array}{c}
51.9469 \\
52.3242 \\
27.482
\end{array}\right) .
$$

The solution of the linear system is: $P_{m}=(3.73968,1.73656,1.14508)$. The distance of $P_{m}$ to $P_{1}$ is 4.27926 , the distance of $P_{m}$ to $P_{2}$ is 3.07179 , the distance of $P_{m}$ to $P_{3}$ is 2.83245 and the distance of $P_{m}$ to $P_{4}$ is 6.23721 . These numerical results have been obtained with Mathematica.

\section{Conclusion}

A coherent derivation of radius and center of the insphere of a general tetrahedron is given. By means of elementary techniques of linear algebra the original linear system for the determination of radius and center of the insphere of a tetrahedron can be broken up to calculate the radius and the coordinates of the center separately. The remaining linear system for the coordinates of the center of the insphere can be solved by multiplying with the inverse of the coefficient matrix, which is found in the course of the treatise. This procedure can readily be applied in the planar case. 


\section{Conflicts of Interest}

The author declares no conflicts of interest regarding the publication of this paper.

\section{References}

[1] https://groups.google.com/forum/\#!topic/sci.math/VsGH4BioURE

[2] Courant, R. (1964) Differential and Integral Calculus, Volume II. Blackie \& Son Limited, London and Glasgow.

[3] Bronshtein, I.N., Semendyayev, K.A., Musiol, G. and Muehlig, H. (2007) Handbook of Mathematics. 5th Edition, Springer, Berlin, Heidelberg, New York.

[4] http://maths.ac-noumea.nc/polyhedr/tetra.htm 